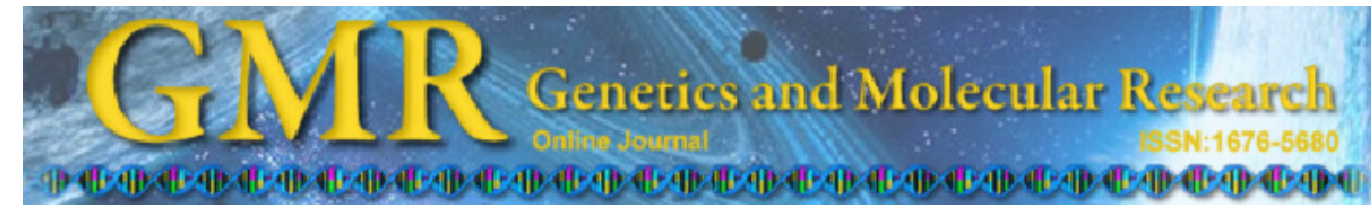

$\underline{\text { Short Communication }}$

\title{
Suitable internal control microRNA genes for measuring miRNA abundance in pig milk during different lactation periods
}

\author{
Y.R. Gu*, Y. Liang*, J.J. Gong, K. Zeng, Z.Q. Li, Y.F. Lei, \\ Z.P. He and X.B. Lv \\ Sichuan Animal Science Academy, Chengdu, Sichuan, China \\ *These authors contributed equally to this study. \\ Corresponding author: X.B. Lv \\ E-mail: lvxuebin@21cn.com
}

Genet. Mol. Res. 11 (3): 2506-2512 (2012)

Received October 7, 2011

Accepted May 20, 2012

Published June 18, 2012

DOI http://dx.doi.org/10.4238/2012.June.18.3

\begin{abstract}
Determination of an optimal set/number of internal control microRNA (miRNA) genes is a critical, but often undervalued, detail of quantitative gene expression analysis. No validated internal genes for miRNA quantitative PCR (q-PCR) in pig milk were available. We compared the expression stability of six porcine internal control miRNA genes in pig milk from different lactation periods ( 1 h, 3 days, 7 days, 14 days, 21 days, and 28 days postpartum), using an EvaGreen q-PCR approach. We found that using the three most stable internal control genes to calculate the normalization factor is sufficient for producing reliable q-PCR expression data. We also found that miRNAs are superior to ribosomal RNA (rRNA) and snRNA, which are commonly used as internal controls for normalizing miRNA q-PCR data. In terms of economic and experimental feasibility, we recommend the use of the three most stable internal control miRNA genes (miR-17, -107 and -103) for calculating the normalization factors
\end{abstract}


for pig milk samples from different lactation periods. These results can be applied to future studies aimed at measuring miRNA abundance in porcine milk.

Key words: Pig; miRNA; Normalization; Milk; Quantitative PCR

\section{INTRODUCTION}

MicroRNAs (miRNAs) are a large class of post-transcriptional regulators that bind to the 3'-untranslated region of mRNAs and play important roles in governing diverse biological processes, including cell proliferation and differentiation, apoptosis, and metabolism (Bartel, 2004). Quantitation of miRNA gene expression levels is an essential step for better understanding their roles in biological processes. Among the technologies used for quantization of miRNA expression, quantitative polymerase chain reaction (q-PCR)-based techniques are particularly attractive for miRNA profiling owing to their unsurpassed sensitivity, cost-effectiveness, and capability for large-scale characterizations of miRNA expression patterns (Nolan et al., 2006; Bartels and Tsongalis, 2009). Producing reliable q-PCR expression data, however, requires that corrections be made for differences between samples that arise from the varying quality and quantity of the starting material, which are often consequence of the method of RNA extraction and complementary DNA (cDNA) synthesis (Stahlberg et al., 2004). Internal control genes are widely used to adjust for these variables, which are not a result of the experimental design.

Only $0.01 \%$ of the total RNA in a sample is composed of miRNAs, and this fraction can vary significantly across samples because the extraction efficiency of miRNA is significantly different from that for much longer RNAs (Davoren et al., 2008; Peltier and Latham, 2008). Therefore, choosing an appropriate set of miRNA genes as internal control genes is crucial, especially for normalizing a set of miRNA q-PCR data; the inappropriate normalization of q-PCR data can lead to incorrect conclusions (Vandesompele et al., 2002; Schaefer et al., 2010). Moreover, the most frequently used internal control genes are ribosomal RNA (rRNA) and small nuclear RNA (snRNA). Only one internal control gene was used in almost all previous reports of various methods for expression profile analysis related to the measurement of miRNA abundance in pigs (Kim et al., 2006; Li et al., 2011). Some debate exists, however, over the use of rRNAs as internal controls, because some studies have shown that they can be expressed at much greater levels than the target gene, making it difficult to quantify a rare miRNA transcript (Bustin, 2000; Vandesompele et al., 2002). Previous studies have also shown that snRNA has poor stability (Peltier and Latham 2008; Chang et al., 2010). Furthermore, the use of a single internal control gene for normalization leads to large errors (Vandesompele et al., 2002; Mortarino et al., 2010). Therefore, the identification of a set of suitable internal control miRNA genes would be valuable for studies investigating the abundance of interesting miRNAs in various developmental stages and samples in pigs.

In this study, we performed a systematic study of a panel of 3 miRNAs, 2 rRNAs, and 1 snRNA using pig milk samples from 6 lactation periods to determine their suitability as internal controls for normalizing miRNA gene expression data. We expect this study to stimulate widespread and in-depth studies of miRNA expression profiles in pig milk. 


\section{MATERIAL AND METHODS}

\section{Sample collection and total RNA extraction}

Pig milk samples were collected at six lactating stages ( 1 h, 3 days, 7 days, 14 days, 21 days, and 28 days) from three female Berkshire pigs after normal delivery. All samples were immediately frozen in liquid nitrogen and stored at $-80^{\circ} \mathrm{C}$ until analysis. Total RNA was extracted using the TRIzol LS Reagent (Invitrogen, USA) from the 18 pig milk samples following the manufacturer protocol. Extracted crude RNA was treated with Deoxyribonuclease I, Amplification Grade (Invitrogen) according to the manufacturer protocol to remove contaminant genomic DNA. The total RNA quantity and purity were analyzed at 260/280 nm using a NanoDrop ND-1000 spectrophotometer (NanoDrop Technologies, USA).

\section{EvaGreen q-PCR assays}

Equal amounts of RNA were reverse transcribed to cDNA using a One Step PrimeScript miRNA cDNA Synthesis Kit (TaKaRa, China), following manufacturer recommendations. The EvaGreen-based q-PCR was performed with the SsoFast EvaGreen Supermix Kit (Bio-Rad, USA) on a CFX96 Real-Time PCR Detection System (Bio-Rad). Each reaction comprised 10 $\mu \mathrm{L}$ SsoFast EvaGreen Supermix, $2 \mu \mathrm{L}$ cDNA, $1.0 \mu \mathrm{L}$ universal reverse primer (TaKaRa) and miRNA-specific forward primer (miRNA) or each gene-specific primer (rRNA and snRNA), and RNA-free water (Ambion, USA) to a total volume of $20 \mu \mathrm{L}$. The real-time PCR program started with $30 \mathrm{~s}$ of denaturation at $98^{\circ} \mathrm{C}$, followed by 45 cycles of $1 \mathrm{~s}$ of denaturation at $98^{\circ} \mathrm{C}, 5$ $\mathrm{s}$ of annealing at $60^{\circ} \mathrm{C}$ for all specific primers (Table 1), and $1 \mathrm{~s}$ of elongation at $65^{\circ} \mathrm{C}$. Fluorescence was measured during the annealing step of each cycle. The specific PCR products were confirmed through melting curve analysis, which verified the presence of one gene-specific peak and the absence of primer dimers. All measurements contained three negative controls, and each sample of each individual was analyzed in triplicate. The cycle threshold $\left(\mathrm{C}_{t}\right)$ value was converted to relative abundance using the $2^{-\Delta \Delta \mathrm{Ct}}$ method for further analysis.

\begin{tabular}{llll}
\multicolumn{2}{c}{ Table 1. Primers used for EvaGreen q-PCR. } \\
\hline Name & GenBank/miRBase accession or reference & Primer sequence $\left(5^{\prime}-3^{\prime}\right)$ & Tm $\left({ }^{\circ} \mathrm{C}\right)$ \\
\hline $5 S$ & AF329851 & F: GCCCGATCTCGTCTGATCT & 60 \\
Ret- $t R N A$ & Xie et al. $(2010)$ & F: CAGCTACAGCACCCGGTATT & 60 \\
miR-17 & MI0008214 & R: CGATCCATCGACCTCTGGGTTATG & 60 \\
miR-103 & MI0002448 & CAAAGTGCTTACAGTGCAGGTAG & 60 \\
miR-107 & MI0002449 & AGCAGCATTGTACAGGGCTATGA & 60 \\
& EU520423 & AGCAGCATTGTACAGGGCTATCA & 60 \\
\hline
\end{tabular}

$\mathrm{Tm}=$ melting temperature

\section{Analysis of expression stability}

The stabilities of six well-known internal control genes were evaluated using the geNorm algorithm (Vandesompele et al., 2002). Gene names and GenBank/miRBase accession 
Nos. are listed in Table 1. We used geNorm to calculate the average expression stability values $(M)$ for a candidate gene and express it as the average pairwise variation $(V)$ for that gene with all other tested candidate genes. Stepwise exclusion of the gene with the highest $M$ value allows ranking of the tested genes according to their expression stability, and the most stable genes are ranked in a way that is insensitive to highly unstable "outliers" in the test group (Vandesompele et al., 2002). To determine the optimal number of internal control genes for normalization, we calculated the pairwise variation $V_{\mathrm{n} / \mathrm{n}+1}$ between each set of 2 sequential normalization factors $\left(\mathrm{NF}_{\mathrm{n}}\right.$ and $\left.\mathrm{NF}_{\mathrm{n}+1}\right)$, reflecting the effect of adding an $(\mathrm{n}+1)$ th candidate gene. A large variation means that the added gene has a significant effect and should be included for calculation of a reliable NF value. The recommended cutoff value for pairwise variation was 0.15 . In addition, the observed trend (of changing $V$ values when using additional genes) can also be equally informative; the optimal number of genes for normalization means having the least pairwise variation.

\section{RESULTS}

The stabilities of the six internal control genes were analyzed using the geNorm algorithm, and their $M$ values are listed in Figure 1. In all surveyed genes, $m i R-17 / m i R-107$ were the most stable genes pairwise $(M=0.275)$, whereas $5 S$ was the least stable gene $(M=0.795)$. Notably, the rRNA and snRNA had the least expression stability and were less suitable than the miRNAs $(m i R-17, m i R-107$, and $m i R-103)$ for use as internal control genes for relative quantification of miRNA expression in pig milk, despite being the most commonly used normalizers in miRNA q-PCR.

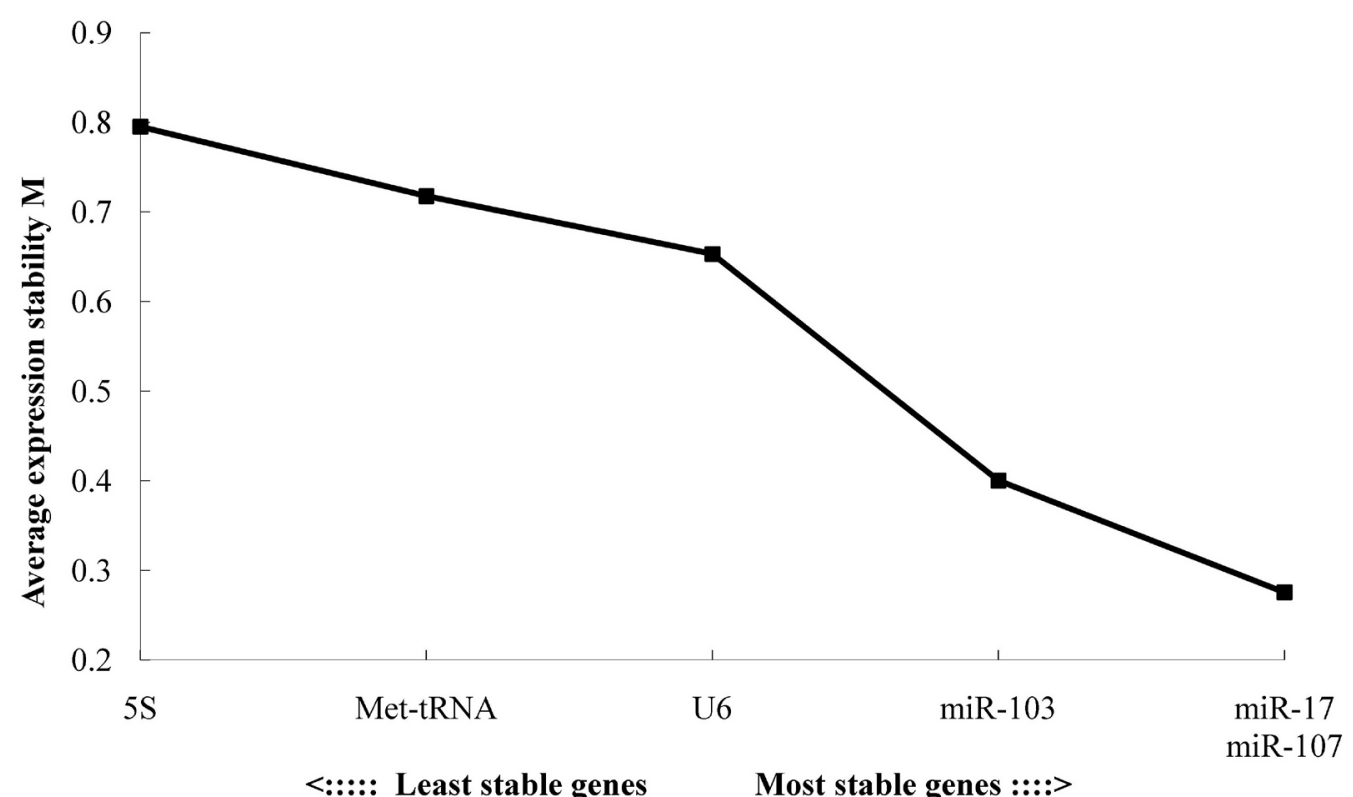

Figure 1. Average expression stability values $(M)$ of six candidate internal control pig genes according to the geNorm algorithm. The gene stability value $M$ is based on the average pairwise variation between all tested genes. The highest $M$ values correspond to the least stable genes, while the lowest $M$ values correspond to the most stable genes. 
We found five $(V=0.144)$ internal control genes in the surveyed pig milk samples with the lowest $V$ values, indicating that the optimal normalization of miRNA expression could be achieved using the top five most stable internal controls (Figure 2). Using excessive numbers of internal control genes for normalization is impractical, however, particularly when studying only a small number of target genes or rare samples that are very difficult to acquire. With these practical considerations taken into account, the use of the three most stable internal control genes for the calculation of the NF was considered acceptable for the majority of experiments. Our results show that the pairwise variations were 0.149 and 0.144 for the three most stable genes and the optimal number of genes, respectively, which are both under the recommended cutoff value. The results also demonstrate that using the three best internal control genes simultaneously is a valid normalization strategy and is sufficient for an accurate normalization of pig milk miRNA q-PCR data.

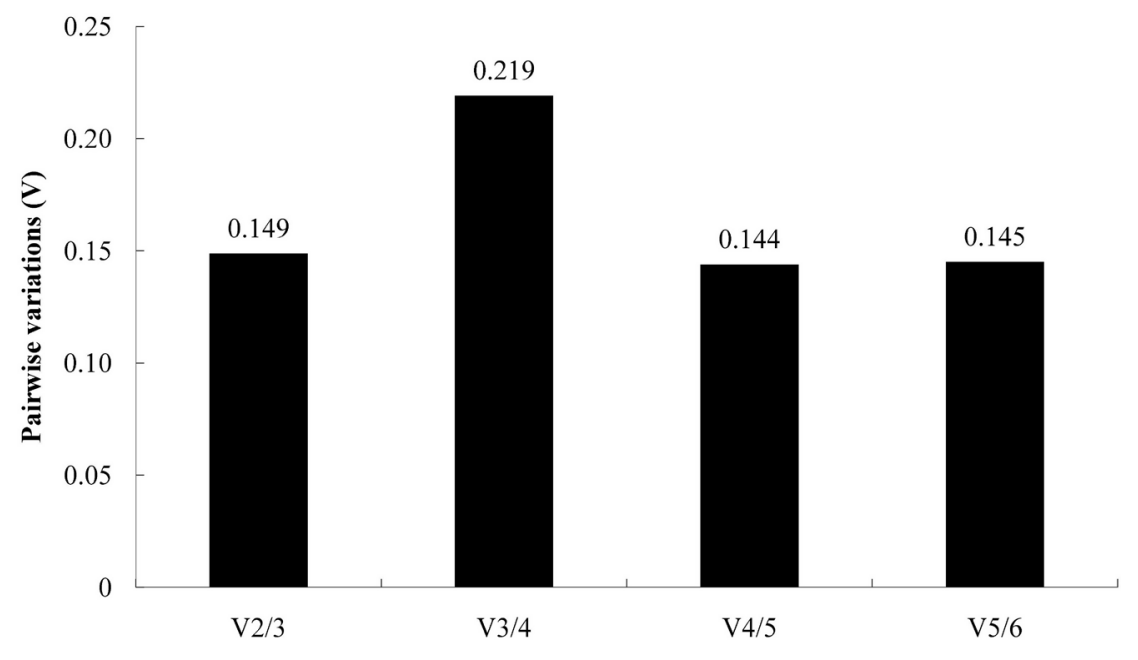

Figure 2. Determination of the optimal number of internal genes for normalization according to the geNorm algorithm. Pairwise variation analysis to determine the number of internal control genes required for accurate normalization was performed in pig milk samples from different lactation periods.

\section{DISCUSSION}

Identifying a specific set of internal control genes is essential for accurate normalization of q-PCR data because even frequently used internal control genes are not always stable under certain conditions. No single control is universal for all experimental conditions, and the use of more than one internal control gene can produce much more accurate and reliable normalization of q-PCR data. Furthermore, more rigorous normalization strategies should be introduced for miRNA q-PCR than for other classes of RNA q-PCR because the low proportion of miRNA in total RNA means that even small changes in miRNA expression may be biologically significant (Davoren et al., 2008; Peltier and Latham, 2008).

The identification and validation of suitable internal control genes for mRNA gene expression analysis has been discussed widely in previous studies (de Kok et al., 2005; Gu et 
al., 2011). Nonetheless, the development of a new set of internal genes for the normalization of miRNA q-PCR data has been long overdue, and no previous report has detailed the identification and validation of suitable internal control genes for normalization in pig milk. Here, we systematically evaluated the reliability of six well-known miRNA internal control genes across 18 pig milk samples during different periods of lactation. Our study shows that $m i R-17, m i R-$ 107 , and miR-103 are optimal candidates for internal controls for research comparing miRNA expression across pig milk samples; these genes showed the most stable expression across all surveyed samples. Moreover, we found that $5 S$ and $U 6$, the most commonly used internal controls for the normalization of miRNA q-PCR data, were not the most stable across all pig milk samples or varying periods of lactation. These results were in accordance with the findings of Peltier and Latham (2008), who have stated that $5 S$ and $U 6$ are unsuitable internal controls for normalizing miRNA gene expression data. Our data also suggest that miRNAs such as $m i R-17$, $m i R-107$, and $m i R-103$ are superior to rRNA and snRNA for normalizing miRNA q-PCR data. It is hypothesized that the tested miRNAs have the same purification properties, are closer in biogenesis progress, have the same sequence characteristics, and use the same assays as the target miRNA. Thus, normalizing genes with internal control genes belonging to the same RNA class is best because they share similar properties. This theory was also supported by a study on normal and cancerous human solid tissues, in which rRNA and snRNA were found to be the least stable in a set of identified miRNA internal controls (Peltier and Latham, 2008).

In summary, our findings provide recommendations for the choice of internal control genes in studies of miRNA expression in pig milk samples. Further customized surveys that focus on different tissue types to create an atlas of various porcine tissues will be invaluable for researchers.

\section{ACKNOWLEDGMENTS}

Research supported by the Open Fund of Sichuan Provincial Key Laboratory of Animal Breeding and Genetics, Sichuan Animal Science Academy (\#SASA2011A01), and the Specialized Research Fund for Animal Breeding Program of Sichuan Province (\#SASA2009YZ001).

\section{REFERENCES}

Bartel DP (2004). MicroRNAs: genomics, biogenesis, mechanism, and function. Cell 116: 281-297.

Bartels CL and Tsongalis GJ (2009). MicroRNAs: novel biomarkers for human cancer. Clin. Chem. 55: 623-631.

Bustin SA (2000). Absolute quantification of mRNA using real-time reverse transcription polymerase chain reaction assays. J. Mol. Endocrinol. 25: 169-193.

Chang KH, Mestdagh P, Vandesompele J, Kerin MJ, et al. (2010). MicroRNA expression profiling to identify and validate reference genes for relative quantification in colorectal cancer. BMC Cancer 10: 173 .

Davoren PA, McNeill RE, Lowery AJ, Kerin MJ, et al. (2008). Identification of suitable endogenous control genes for microRNA gene expression analysis in human breast cancer. BMC Mol. Biol. 9: 76.

de Kok JB, Roelofs RW, Giesendorf BA, Pennings JL, et al. (2005). Normalization of gene expression measurements in tumor tissues: comparison of 13 endogenous control genes. Lab. Invest. 85: 154-159.

Gu YR, Li MZ, Zhang K, Chen L, et al. (2011). Evaluation of endogenous control genes for gene expression studies across multiple tissues and in the specific sets of fat- and muscle-type samples of the pig. J. Anim. Breed. Genet. 128: 319-325.

Kim HJ, Cui XS, Kim EJ, Kim WJ, et al. (2006). New porcine microRNA genes found by homology search. Genome 49: 1283-1286. 
Li G, Li Y, Li X, Ning X, et al. (2011). MicroRNA identity and abundance in developing swine adipose tissue as determined by Solexa sequencing. J. Cell Biochem. 112: 1318-1328.

Mortarino M, Gioia G, Gelain ME, Albonico F, et al. (2010). Identification of suitable endogenous controls and differentially expressed microRNAs in canine fresh-frozen and FFPE lymphoma samples. Leuk. Res. 34: 1070-1077.

Nolan T, Hands RE and Bustin SA (2006). Quantification of mRNA using real-time RT-PCR. Nat. Protoc. 1: 1559-1582.

Peltier HJ and Latham GJ (2008). Normalization of microRNA expression levels in quantitative RT-PCR assays: identification of suitable reference RNA targets in normal and cancerous human solid tissues. RNA 14: 844-852.

Schaefer A, Jung M, Miller K, Lein M, et al. (2010). Suitable reference genes for relative quantification of miRNA expression in prostate cancer. Exp. Mol. Med. 42: 749-758.

Stahlberg A, Hakansson J, Xian X, Semb H, et al. (2004). Properties of the reverse transcription reaction in mRNA quantification. Clin. Chem. 50: 509-515.

Vandesompele J, De Preter K, Pattyn F, Poppe B, et al. (2002). Accurate normalization of real-time quantitative RT-PCR data by geometric averaging of multiple internal control genes. Genome Biol. 3: RESEARCH0034.

Xie SS, Huang TH, Shen Y, Li XY, et al. (2010). Identification and characterization of microRNAs from porcine skeletal muscle. Anim. Genet. 41: 179-190. 